\title{
A systematic study of the isothermal crystallization of the mono-alcohol $n$-butanol monitored by dielectric spectroscopy
}

Jensen, Mikkel Hartmann; Hecksher, Tina; Niss, Kristine; Alba-Simionesco, Christiane

Published in:

Journal of Chemical Physics

DOI:

$10.1063 / 1.4931807$

Publication date:

2015

Document Version

Publisher's PDF, also known as Version of record

Citation for published version (APA):

Jensen, M. H., Hecksher, T., Niss, K., \& Alba-Simionesco, C. (2015). A systematic study of the isothermal crystallization of the mono-alcohol n-butanol monitored by dielectric spectroscopy. Journal of Chemical Physics, 143, [134501]. https://doi.org/10.1063/1.4931807

\section{General rights}

Copyright and moral rights for the publications made accessible in the public portal are retained by the authors and/or other copyright owners and it is a condition of accessing publications that users recognise and abide by the legal requirements associated with these rights.

- Users may download and print one copy of any publication from the public portal for the purpose of private study or research.

- You may not further distribute the material or use it for any profit-making activity or commercial gain.

- You may freely distribute the URL identifying the publication in the public portal.

Take down policy

If you believe that this document breaches copyright please contact rucforsk@kb.dk providing details, and we will remove access to the work immediately and investigate your claim. 


\section{A|P| $\begin{aligned} & \text { The Journal of } \\ & \text { Chemical Physics }\end{aligned}$}

\section{A systematic study of the isothermal crystallization of the mono-alcohol n-butanol monitored by dielectric spectroscopy}

M. H. Jensen, C. Alba-Simionesco, K. Niss, and T. Hecksher

Citation: The Journal of Chemical Physics 143, 134501 (2015); doi: 10.1063/1.4931807

View online: http://dx.doi.org/10.1063/1.4931807

View Table of Contents: http://scitation.aip.org/content/aip/journal/jcp/143/13?ver=pdfcov

Published by the AIP Publishing

\section{Articles you may be interested in}

Vibrational and structural properties of amorphous n-butanol: A complementary Raman spectroscopy and Xray diffraction study

J. Chem. Phys. 138, 214506 (2013); 10.1063/1.4808159

Diluting the hydrogen bonds in viscous solutions of $\mathrm{n}$-butanol with $\mathrm{n}$-bromobutane: A dielectric study

J. Chem. Phys. 128, 154520 (2008); 10.1063/1.2903403

Dielectric relaxation and crystallization of nanophase separated 1-propanol-isoamylbromide mixture

J. Chem. Phys. 127, 094507 (2007); 10.1063/1.2761898

Spontaneous transformation of water's high-density amorph and a two-stage crystallization to ice VI at $1 \mathrm{GPa}$ :

A dielectric study

J. Chem. Phys. 120, 11662 (2004); 10.1063/1.1747946

Dielectric relaxation of hydrogen bonded liquids: Mixtures of monohydric alcohols with n-alkanes

J. Chem. Phys. 115, 4186 (2001); 10.1063/1.1389293

\section{AlP $\mid$ APL Photonics}

APL Photonics is pleased to announce Benjamin Eggleton as its Editor-in-Chief

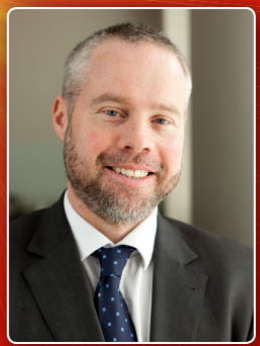




\title{
A systematic study of the isothermal crystallization of the mono-alcohol n-butanol monitored by dielectric spectroscopy
}

\author{
M. H. Jensen, ${ }^{1,2}$ C. Alba-Simionesco, ${ }^{2}$ K. Niss,${ }^{1}$ and T. Hecksher ${ }^{1, a)}$ \\ ${ }^{1}$ Department of Sciences, DNRF Centre Glass and Time, IMFUFA, Roskilde University, P.O. Box 260, \\ DK-4000 Roskilde, Denmark \\ ${ }^{2}$ Laboratoire Léon Brillouin, CNRS CEA -UMR 12, DSM IRAMIS LLB CEA Saclay, \\ 91191 Gif-sur-Yvette Cedex, France
}

(Received 23 June 2015; accepted 14 September 2015; published online 2 October 2015)

\begin{abstract}
Isothermal crystallization of the mono-hydroxyl alcohol $n$-butanol was studied with dielectric spectroscopy in real time. The crystallization was carried out using two different sample cells at 15 temperatures between $120 \mathrm{~K}$ and $134 \mathrm{~K}$. Crystallization is characterized by a decrease of the dielectric intensity. In addition, a shift in relaxation times to shorter times was observed during the crystallization process for all studied temperatures. The two different sample environments induced quite different crystallization behaviors, consistent and reproducible over all studied temperatures. An explanation for the difference was proposed on the background of an Avrami analysis and a Maxwell-Wagner analysis. Both types of analysis suggest that the morphology of the crystal growth changes from a higher dimension to a lower at a point during the crystallization. More generally, we conclude that a microscopic interpretation of crystallization measurements requires multiple probes, sample cells, and protocols. (C) 2015 AIP Publishing LLC. [http://dx.doi.org/10.1063/1.4931807]
\end{abstract}

\section{INTRODUCTION}

All liquids can be supercooled. ${ }^{1-3}$ In fact, crystallization rarely takes place exactly at the melting temperature upon cooling, because the crystal nuclei formed dissolve before they grow to a stable size. ${ }^{4}$ Some liquids, like water, crystallize readily at moderate supercooling and need fast quenching below the melting temperature in order to avoid crystallization. ${ }^{5}$ In fact, water is so prone to crystallization that there is a broad range of temperatures - the "no-man's land"- where the supercooled liquid state is inaccessible for the bulk liquid. ${ }^{6,7}$ Other liquids, like the prototype glass-former glycerol, supercool easily and require a careful protocol to crystallize. ${ }^{8}$ But the true thermodynamic equilibrium state for all supercooled liquids and glasses is unarguably the crystal, and thus, crystallization is their eventual inevitable fate.

For many applications, the life-time of the glassy or meta-stable liquid state is a key issue and the ability to predict and control crystallization properties is desired. But understanding the crystallization process is also interesting from a fundamental point of view. Crystallization studies are however difficult to carry out in a controlled and reproducible manner, because many factors influence the initiation and course of crystallization, such as sample preparation, thermal history, presence of impurities, and container geometry. ${ }^{8-10}$

We present here a thorough dielectric study of the crystallization process in supercooled $n$-butanol considering a minimum of external parameters. Dielectric spectroscopy is a convenient and fairly common probe for studying crystallization kinetics in real time, see, e.g., Refs. 11-16. The sign of crystallization is a decrease in intensity of

\footnotetext{
a) tihe@ ruc.dk
}

the signal, and the crystal concentration of the sample is often obtained by assuming that the relaxation strength is proportional to the volume fraction of liquid in the sample. ${ }^{17-19}$ But the microscopic interpretation of dielectric spectra is not straightforward, ${ }^{20}$ especially when studying a heterogeneous mixture.

Supercooled $n$-butanol has an intense low-frequency dielectric signal — the so-called Debye-process — characteristic of many monohydroxyl alcohols. ${ }^{21}$ It also exhibits a slow crystallization process upon reheating after a rapid quench below $T_{g} .{ }^{22,23}$ At $10 \mathrm{~K}$ above the glass transition temperature, the crystallization can take several days to finish. These two properties, a large dielectric signal and slow crystallization, make $n$-butanol an ideal candidate for monitoring isothermal crystallization in real time by dielectric spectroscopy. Crystallization of $n$-butanol has previously been studied with x-ray diffraction, ${ }^{22,23}$ Raman spectroscopy, ${ }^{24,25}$ calorimetric methods, ${ }^{26}$ and phase contrast microscopy. ${ }^{27}$ The focus of these studies was primarily on the curious "aborted crystallization" at temperatures close to the glass transition temperature. In the present study, our main focus is not the mechanism of the aborted crystallization, but rather to explore the potential of dielectric spectroscopy for monitoring crystallization on a well-studied crystallizing substance, varying only a small subset of the parameters that influence the crystallization process.

Thus, we used two different dielectric measuring cells with different geometries and different electrode materials and studied a range of temperatures close to $T_{g}$. Measurements from different cells give information about what observations are intrinsic to the sample and what effects should be ascribed to the macroscopic boundary conditions. Measurements at many different temperatures evidently give the temperature 
dependence of crystallization and relaxation times, but in addition, they serve as a consistency and reproducibility check.

\section{EXPERIMENT AND MATERIALS}

All measurements were carried out in the same experimental setup (described in detail in Ref. 28), including a custom-built nitrogen cryostat capable of keeping the temperature stable within $50 \mathrm{mK}$ over weeks. Two different sample cells were used. Cell A is a 22-layered gold-plated parallel plate capacitor with $0.2 \mathrm{~mm}$ between each set of plates and a geometric capacitance of $65 \mathrm{pF}$. Each plate is a semicircle which can be rotated to overlap each other (identical to the capacitors used in old radios). Cell B is a parallel plate capacitor with circular beryllium-copper plates separated by $50 \mu \mathrm{m}$ sapphire spacers and a geometric capacitance of $17.6 \mathrm{pF}$. The cells are sketched in Fig. 1.

The sample $n$-butanol $\left(T_{m}=183 \mathrm{~K}, T_{g} \approx 110 \mathrm{~K}\right)$ was purchased from Sigma Aldrich's at $>99.9 \%$ purity and used without further purification.

The same protocol was followed for each crystallization measurement; a new sample was quenched to $85 \mathrm{~K}$ (roughly $25 \mathrm{~K}$ below $T_{g}$ ) and kept at this temperature for (at least) $120 \mathrm{~min}$, then heated to the target temperature where the crystallization process was followed. The heating took less than $5 \mathrm{~min}$. Frequency scans were made continuously as soon as heating from $85 \mathrm{~K}$ initiated and until no further changes in the spectrum occurred. The frequency range of the scans was adjusted for each temperature to keep the scan as short - and thereby as fast - as possible, while still keeping both $\epsilon_{\infty}$ and $\epsilon_{s}$ in the frequency window. The sample cells were emptied and cleaned between each measurement.

Isothermal crystallization was followed at 15 different temperatures between 134 and $120 \mathrm{~K}$. The exact temperatures in Kelvin are 134, 133, 132, 131, 130, 129, 128.5, 127.5, 126, $125,124,123,122.5,121$, and 120 . The $133 \mathrm{~K}$ measurement has only been done with cell $\mathrm{A}$, while the $132 \mathrm{~K}$ measurement has only been done with cell B. For reference, a fully crystallized sample was made by quenching to $85 \mathrm{~K}$ and reheating to $170 \mathrm{~K}$, and a spectrum of the crystal was measured at all temperatures included in the study.

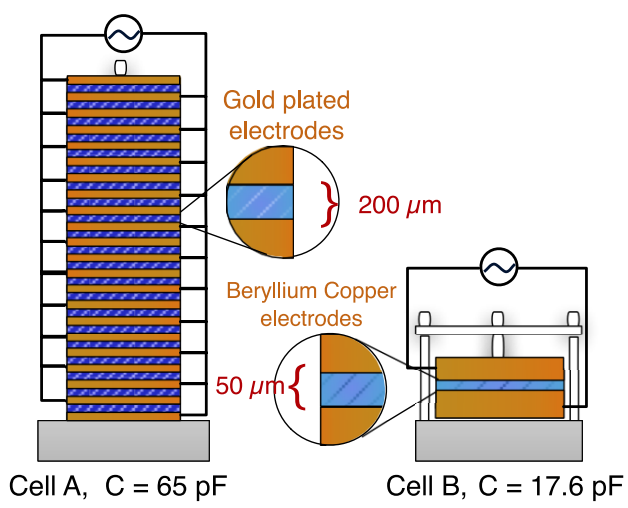

FIG. 1. Schematic drawings of the two cells used for the crystallization studies. Cell A is a multi-layer capacitor with gold plated electrodes (variable capacitor type used in old fashioned radios) with a geometric capacitance of $65 \mathrm{pF}$. Cell B is a two-plate capacitor with beryllium copper electrodes and $50 \mu \mathrm{m}$ sapphire spacers. Cell $\mathrm{B}$ has a geometric capacitance of $17.6 \mathrm{pF}$.

\section{EXPERIMENTAL RESULTS AND DATA ANALYSIS}

\section{A. Phenomenological fits of the spectra}

In $n$-butanol, there are three visible processes - Debye, alpha, and beta processes - in the measured frequency window, see Fig. 2(a). The crystallization, signaled by a decrease of relaxation strength, also induces a shift in the loss peak for the three processes. To quantify how the crystallization influences each of these processes, we fitted
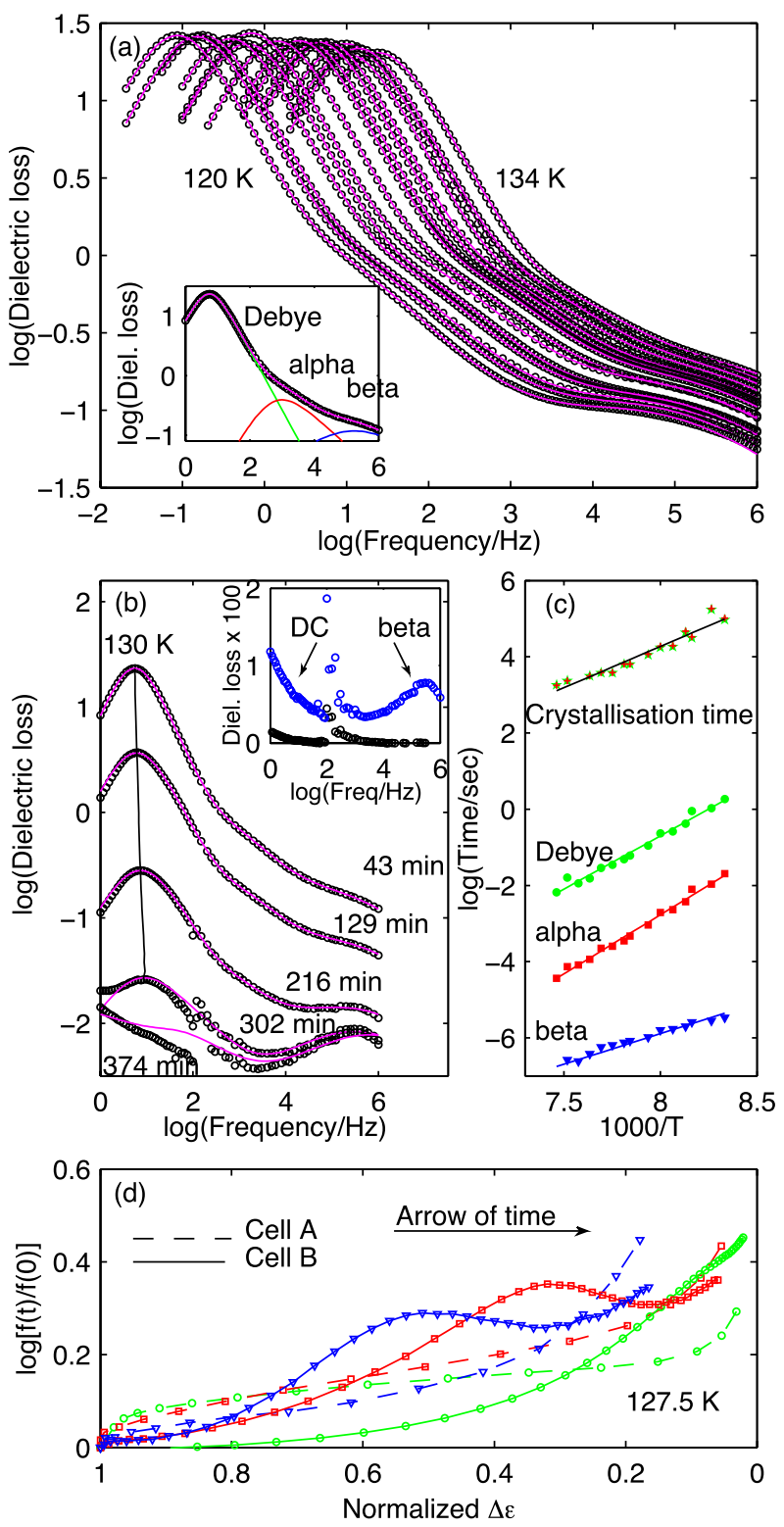

FIG. 2. Fitted spectra and time and temperature dependence of the fitting parameters. (a) The first measurement at every temperature, presumably before crystallization initiates. The magenta lines are fits described in the text. The inset shows the three relaxation processes constituting the fit: the Debye (green), alpha (red), and beta (blue) processes. (b) Selected spectra during crystallization at $130 \mathrm{~K}$, using cell $\mathrm{B}$. Again, magenta lines are fits. The inset shows the dielectric loss of the last measurement (blue), together with the measurement of the full crystal (black), on a linear scale. The "spike" at $100 \mathrm{~Hz}$ is an experimental artifact deriving from a non-perfect match between two voltmeters. (c) The fitted relaxation times together with the crystallization time. The lines are linear fits. (d) Parameterised plot of the logarithm of the normalized relaxation frequency $(\log \{f(t) / f(0)\}$ with $f=1 / \tau)$, against the normalized relaxation strength $\Delta \epsilon$, for each of the three processes. The two cells display quite different behavior during crystallization. 
the spectra to a sum of three relaxation processes. Since the processes are not well separated, we aimed at limiting the number of free fitting parameters by the following procedure: First, the Debye process is fitted by a Cole-Cole function. The Debye process broadens during the crystallization, and thus, a pure exponential function would not give a good fit. The result of the Debye fit is then subtracted from the data and the alpha and beta processes are fitted simultaneously as a sum. The beta process is fitted to a Cole-Cole function with a fixed shape parameter, $\beta=0.45$. The alpha process is fitted to a dielectric version of the Extended Bell (EB) model (see Ref. 29) in which the dielectric constant is given by

$$
\epsilon_{\mathrm{EB}}=\epsilon_{\infty}+\frac{\Delta \epsilon}{1+\frac{1}{\left(1+i \omega \tau_{\alpha}\right)^{-1}+k_{\alpha}\left(i \omega \tau_{\alpha}\right)^{-\alpha}}},
$$

where $\epsilon_{\infty}$ is the high-frequency plateau of the real part, $\Delta \epsilon$ is the relaxation strength, $\tau_{\alpha}$ is the relaxation time, $k_{\alpha}$ controls the width of the peak, and $\alpha$ gives the high-frequency power law behavior of the alpha peak. This model for the alpha relaxation gives good fits even when the shape parameters are fixed such that only the relaxation strength and relaxation time are fitted. The slope parameter was fixed to $\alpha=0.5^{30}$ and the width parameter to $k_{\alpha}=1$. The Cole-Davidson function resulted in poorer fits, even with the shape parameter varying freely. In total, we fitted the imaginary part with seven parameters: a relaxation time, $\tau_{x}$, and a relaxation strength, $\Delta \epsilon_{x}$, for each process ( $x$ denoting Debye, alpha, or beta), and a broadening parameter for the Debye process.

The procedure gives excellent fits over the frequency range explored as shown in Fig. 2(a), where the first dielectric spectrum at every annealing temperature is shown as well as the fits resulting from the described fitting procedure. The inset shows a spectrum at $130 \mathrm{~K}$ with each of the individual fitted relaxation processes.

The same fitting procedure was applied to the isothermal crystallization spectra to study the temporal evolution of the three processes during crystallization. The resulting fits are illustrated in Fig. 2(b) showing a subset of the measured spectra at $130 \mathrm{~K}$ using cell $\mathrm{B}$. As the crystallization proceeds, the strength of the Debye and alpha relaxation processes decreases continuously to disappear entirely by the termination of the crystallization process. This is also to be expected, since there should be no large-scale rearrangement of the molecules in the crystal. The beta relaxation process, however, remains active at the termination of the crystallization process. The inset of Fig. 2(b) shows the last scan at $130 \mathrm{~K}$ (where no further changes in the spectrum occurred) together with the spectrum of the fully crystallized sample at the same temperature. For the full crystal, the signal has virtually vanished, while the last scan of $130 \mathrm{~K}$ still displays dynamical features, thus clearly demonstrating that the crystallization process stops before the sample is fully crystallized and that there is still some molecular mobility left, as reported in Refs. 22-27. The results from the fitting routine establish the general behavior of the spectra during the crystallization, but we refrain from analyzing the finer details, especially towards the end of the crystallization process, where Debye and alpha processes have vanished and the fits become unreliable (see, e.g., the lowest curve in Fig. 2(b)).
Using the relaxation strength, $\Delta \epsilon$, as an indicator of the degree of crystallinity, we define a characteristic crystallization time as the time for $\Delta \epsilon$ to decay to half of its initial value. Figure 2(c) shows the crystallization time derived from both Debye and alpha relaxation strengths as a function of inverse temperature, and it is evident that the two measures are identical within the accuracy of our measurements.

Along with the crystallization time, we show the relaxation times obtained from the fits to the first (uncrystallized) spectrum. Clearly, the Debye, alpha, and beta processes as well as the crystallization process are all slowed down with decreasing temperature, and consequently, the different characteristic time scales would all appear to be correlated (at least in this temperature range), but it does not necessarily imply any causation. For the studied temperature span, all the shown time scales are Arrhenius within the noise, although with very different pre-factors.

Focusing now on how the two sample environments influence the crystallization process, we define the relaxation frequency as the inverse of the fitted relaxation times, $f=1 / \tau$. The evolution of both the relaxation strength and relaxation frequency differs for the two cells. Fig. 2(d) shows a parameterised plot of the logarithm of the fitted relaxation frequency (normalized to the initial value) as a function of the fitted normalized relaxation strength for each of the three processes from measurements at $127.5 \mathrm{~K}$ with both cells $\mathrm{A}$ and $\mathrm{B}$. In cell $\mathrm{A}$, there is a shift in the relaxation frequency, $f$, quite early in the crystallization process, then it remains relatively unchanged for all three relaxation processes until an increase sets in again towards the end of the crystallization. In cell $\mathrm{B}$, the shift in relaxation frequency is more gradual. For the Debye process, the shift is monotonous, but for the alpha and the beta processes, the shift displays a non-monotonous behavior. The curve peaks in Fig. 2(d) occur roughly the same waiting time for the alpha and beta processes.

The different progresses of the crystallization process for the two cells suggest a macroscopic/mesoscopic rather than microscopic explanation since a slight difference in sample geometries is not expected to affect the behavior of individual molecules.

The full set of fitted parameters normalized to the initial value is shown as a function of waiting time in Fig. 3. The colors of the curves indicate the temperature with blue being the lowest $(120 \mathrm{~K})$ and red being the highest (134 K). In both cells, lower temperatures lead to longer crystallization times, as was shown in Fig. 2(c).

For cell A (Fig. 3(a)), the temporal evolution of the relaxation strengths for each of the three processes appears similar, except at long waiting times, where the beta relaxation strength levels off at $\sim 0.08$ instead of decaying all the way to zero. The final level for the beta relaxation is marked by a dashed line in the third panel of Fig. 3(a). For cell B (Fig. 3(e)), the Debye and alpha relaxation strengths follow each other until roughly halfway through the crystallization, where a shoulder emerges in the alpha relaxation strength curve, which then proceeds like a two-step relaxation. As in cell $\mathrm{A}$, the beta relaxation strength does not decay to zero and levels off at the same value as for cell A. Comparing Figs. 3(a) and 3(e), we see that the curves for cell B are significantly less 

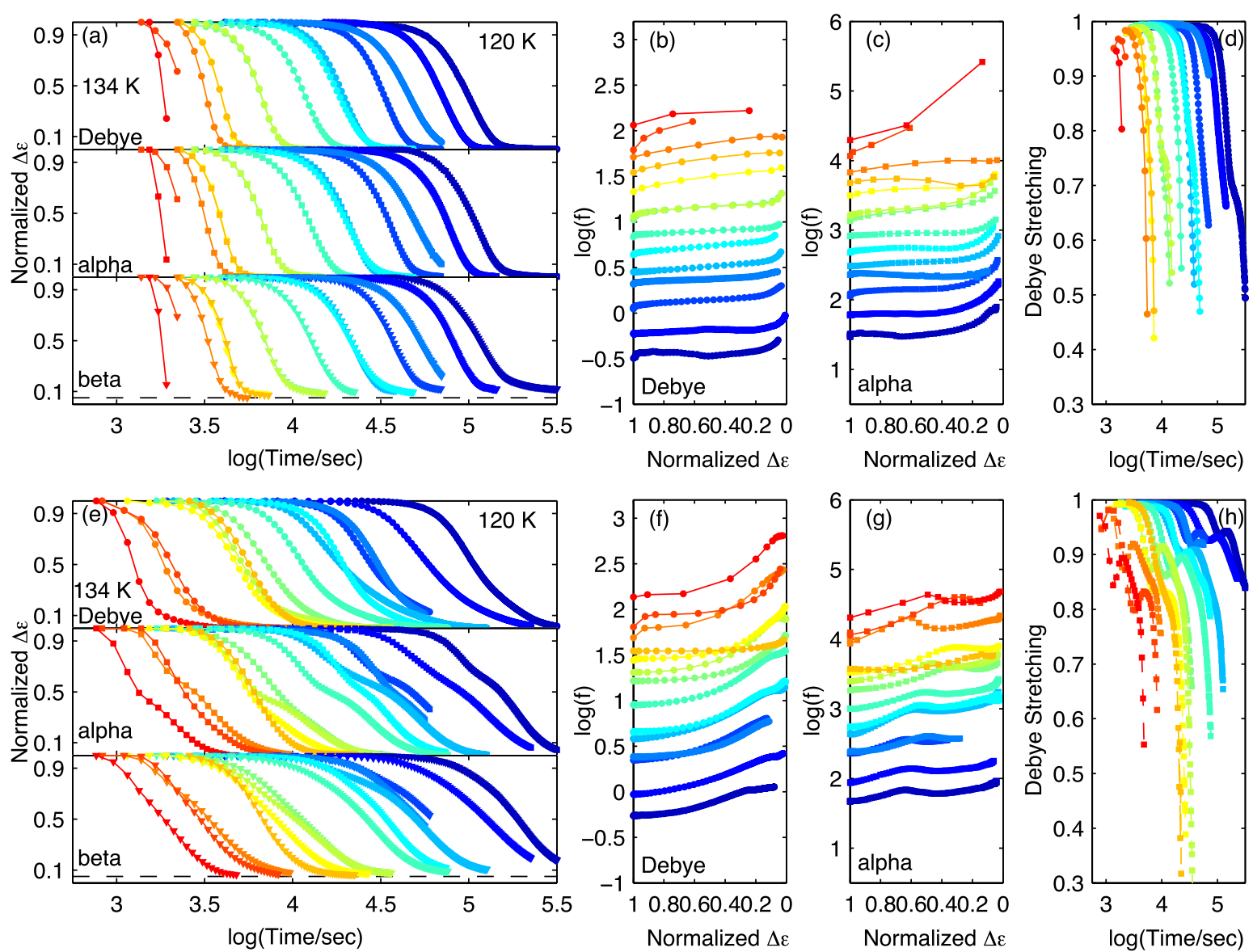

FIG. 3. Parameters for the fitted isothermal crystallization spectra of $n$-butanol for temperatures between $120 \mathrm{~K}$ and $134 \mathrm{~K}$. The colors of the curves indicate the temperatures with blue being the lowest temperature and red the highest. $133 \mathrm{~K}$ has only been done with cell A while $132 \mathrm{~K}$ has only been done with cell $\mathrm{B}$. Notice that the crystallization process takes longer to finish in cell B. (a), (e) The normalized relaxation strengths defined as $\Delta \epsilon(t) / \Delta \epsilon(0)$ of the Debye, alpha, and beta processes for cells A (a) and B (e) as a function of logarithmic time. (b), (c), (f), (g) The logarithm of the relaxation frequency $(f=1 / \tau)$ as a function of the normalized relaxation strength for cell A (b), (c) and cell B (f), (g). (d), (h) The broadening parameter of the Debye process. All fitted values start at 1 (corresponding to a pure exponential) and then gradually drop to $\sim 0.5$. For cell A (d), the broadening is monotonous while for cell B (h), there is a local minimum followed by a maximum for all studied temperatures.

compressed than the corresponding curves for cell A, which means that crystallization proceeds at a consistently slower rate in cell B compared to cell A. Consequently, our definition of crystallization time may give roughly the same for the two cells, but the time it takes before crystallization stops is much longer in cell $\mathrm{B}$.

Figures 3(b), 3(c), 3(f), and 3(g) show the logarithm of relaxation frequencies as a function of normalized relaxation strength. The relaxation frequency of the beta process is not shown, because it does not vary in a systematic way, making further interpretation unjustified. In cell $\mathrm{A}$, the general behavior is that the relaxation frequencies have a slight shift to higher frequencies at the onset of the crystallization, but only increase a little during the remainder of the crystallization. In cell B, the relaxation frequency of Debye and alpha processes does not change in the beginning of the crystallization process, but shifts gradually to higher frequencies. A "bump" occurs in alpha relaxation strength around a normalized relaxation strength of 0.5, showing that the behavior observed in Fig. 2(d) is general.

Last, Figs. 3(d) and 3(h) show the broadening parameter of the Debye process as a function of time. All curves start at the value 1 (corresponding to a pure exponential) and then decay to around 0.5 by the end of the crystallization. Again, we see curve shapes that are similar for measurements in the same cell at all temperatures, but differences between the two cells: in cell $\mathrm{A}$, the gradual broadening is monotonous, while in cell $\mathrm{B}$, we first see a broadening, then narrowing and finally a broadening again. A non-monotonous behavior was also seen in the time evolution of the relaxation frequency of the alpha process.

The differences between the two cells are thus reproduced for all the studied temperatures. One possible mesoscopic explanation for the observed difference in the evolution of the relaxation strength and relaxation times for the two cells could be that the two cells induce different kinds of crystal growth.

\section{B. Maxwell-Wagner (MW) analysis}

For heterogeneous material, one can normally assume additivity in signal from different domains in the sample. However, for dielectric spectroscopy, this is in general not the case. ${ }^{31}$ A difference in the conductivity of the different domains in the material leads to build-up of charges at the interfaces between domains. This gives rise to a polarization effect known as MW polarization. ${ }^{20,32}$ 
In the present case, the heterogeneity is caused by the formation of crystallites in the sample. As domains of crystal grow in the liquid, the dielectric constant for the composite will change. The details of the change will depend on the difference between the dielectric constant of the liquid $\left(\epsilon_{l}\right)$, and that of the crystal $\left(\epsilon_{c}\right)$, the shape of the crystal domain, and the volume fraction taken up by the crystal.

The two simplest cases of crystal domains growing in the liquid are that of a crystal layer growing from one (or both) of the electrodes, a heterogeneous nucleation picture, and the case of crystal spheres in a liquid matrix, a homogeneous nucleation picture.

In the first case, no approximation is involved in deriving the expression for the composite dielectric constant. The two materials (liquid and crystal) in a layered construction are simply modeled by two capacitors connected in series, $C_{\text {tot }}$ $=\left(1 / C_{1}+1 / C_{2}\right)^{-1}$. Thus, the resulting composite dielectric constant is given by ${ }^{31}$

$$
\begin{aligned}
\epsilon_{\text {comp }} & =\frac{d_{c}+d_{l}}{d_{c} / \epsilon_{c}+d_{l} / \epsilon_{l}} \\
& =\frac{\epsilon_{c} \epsilon_{l}}{\left(1-\phi_{\text {slab }}\right) \epsilon_{c}+\phi_{\text {slab }} \epsilon_{l}},
\end{aligned}
$$

where $\phi_{\text {slab }}=d_{c} /\left(d_{c}+d_{l}\right)$ is the relative thickness of the crystal layer. Since $\epsilon_{l}$ and $\epsilon_{c}$ can be measured independently, this model has one free parameter (assuming the distance between the electrodes is fixed, or equivalently that total thickness of crystal and liquid layer is unchanged during crystallization).

Inserting the measured spectrum of $\epsilon_{l}$ (at time $t=0$ before crystallization initiates) and $\epsilon_{c}$ (the spectrum of the fully crystallized sample), this model produces a shift in the peak frequency, but it overestimates the corresponding decrease in relaxation strength. Thus, the model is unable to capture the observed crystallization behavior; so, this scenario alone is not sufficient to explain what we observe.

In the case of crystal domains dispersed in a liquid, a mean-field approximation is used to arrive at the composite dielectric constant ${ }^{33}$

$$
\epsilon_{\mathrm{comp}}=\epsilon_{l} \frac{2 \epsilon_{l}+\epsilon_{c}-2 \phi\left(\epsilon_{l}-\epsilon_{c}\right)}{2 \epsilon_{l}+\epsilon_{c}+\phi\left(\epsilon_{l}-\epsilon_{c}\right)},
$$

where $\phi$ is the concentration of the crystal domains. This model also contains a single fitting parameter, $\phi$. Inserting the measured spectra of $\epsilon_{c}$ and $\epsilon_{l}$ in Eq. (3) does not produce a frequency shift. Consequently, this model cannot account for what we observe either.

Instead, we propose to combine the two models such that a crystal layer is growing from the electrodes, while spherical crystallites are forming in the remaining liquid, see Fig. 4. This is modeled by combining Eqs. (2) and (3) such that $\epsilon_{l}$ in Eq. (2) is given by the composite dielectric constant from Eq. (3). This model has two parameters: the relative thickness of the crystal layer, $\phi_{\text {slab, }}$, and the concentration of crystal spheres in the liquid, $\phi$.

The mean field approximation (Eq. (3)) is only accurate up to $\phi \approx 0.2,{ }^{20}$ but we allow $\phi$ to go somewhat higher. Accuracy is lost when pushing the limits of the mean field approximation, but it could still give an indication of the overall behavior,

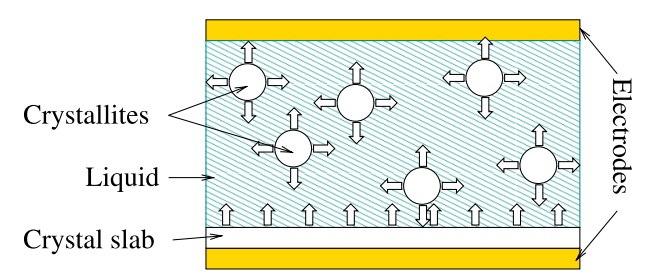

FIG. 4. Cartoon of the combined heterogeneous and homogeneous crystallization. The growth is indicated by the arrows.

especially when it is used in combination with Eq. (2), which is exact. In the combined model, the volume fraction of spheres in the total volume never gets close to 1 (see Fig. 5).

Examples of fits to isothermal crystallization spectra for cell A and cell B are shown in Figs. 5(a) and 5(b), respectively. The fits are focused on the Debye process by only fitting to the points within the two dotted lines. Having two fitting parameters gives sufficient flexibility to account for both the decrease in relaxation strength as well as the change in peak frequency of the Debye peak. However, the combined model does not adequately explain the behavior of the entire spectrum; it does not capture the behavior of the alpha and beta relaxation processes during the crystallization or the broadening of the Debye process. Despite these limitations, the models ability to describe the behavior of the Debye relaxation
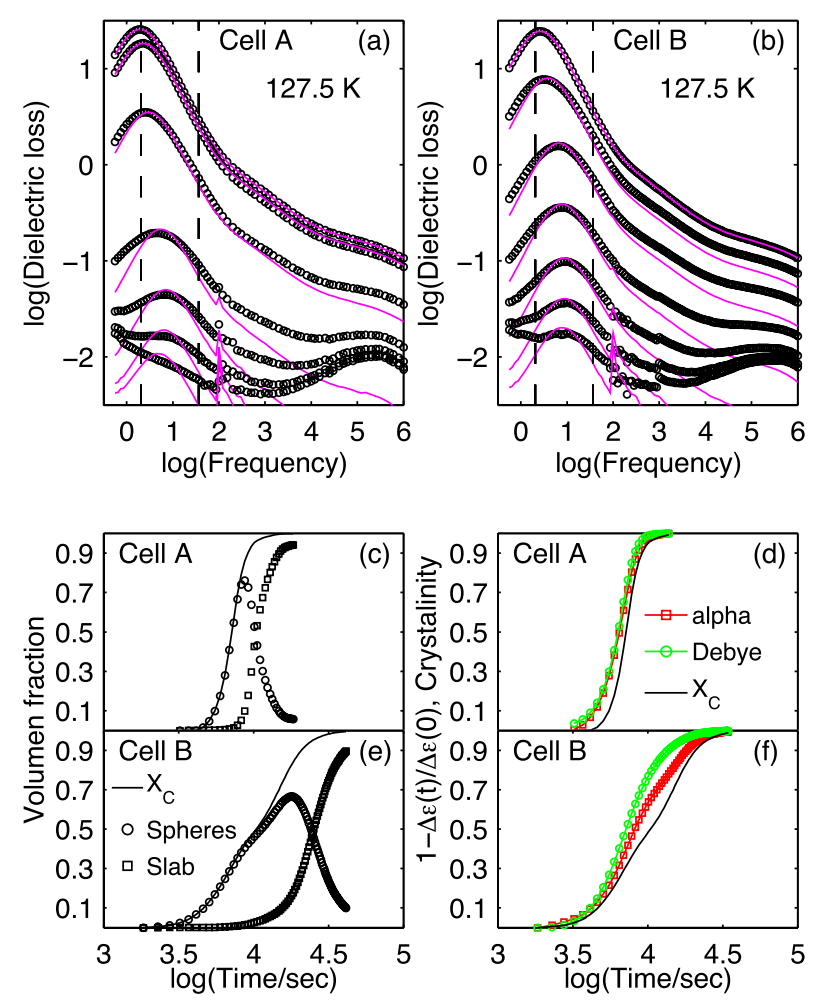

FIG. 5. The measurements shown in Fig. 2 analyzed using the MaxwellWagner (MW) approach described in Section III B. (a) and (b) show a selection of curves together with fits (magenta lines) for cell A (a) and cell B (b). The dashed vertical lines mark the frequency interval used for the fit. (c) and (e) show the volume fraction taken up by the spherical crystallites (circles) and the crystal slab (squares) as a function of time for cell A (c) and cell B (e). The solid line is the total crystal volume fraction. (d) and (f) Total crystal volume fraction from the MW fit (black line) as well as the normalized relaxation strength of the Debye process (in green) and alpha process (in red) as a function of time for cell A (d) and cell B (f). 
strength and peak position may be used to shed some light on the observed phenomena.

The total crystallized volume fraction according to the model can be calculated as $X_{c}=\left(1-\phi_{\text {slab }}\right) \phi+\phi_{\text {slab }}$ and the volume fraction taken up by the spheres alone as $X_{\text {sphere }}$ $=\left(1-\phi_{\text {slab }}\right) \phi$. Figures 5(c) and 5(e) show the volume fraction of the crystal layer and the spherical crystallites as well as the total crystal volume fraction. For both cells, it seems that the onset of crystallization is dominated by nucleation and growth of crystal spheres, and when a large fraction of the sample has crystallized $\left(X_{c} \sim 70 \%-80 \%\right)$, the slab growth takes over. However, the growth of crystal spheres starts earlier but proceeds at a slower rate in cell B compared to cell A. This difference in crystallization behavior in the two cells is reproduced at all temperatures (see Fig. 6).

The total degree of crystallinity as calculated from the MW fits is plotted together with the normalized Debye and alpha relaxation strengths for cell A in Fig. 5(d) and for cell $B$ in Fig. 5(f). Again, the curves are clearly different for the two cells; in cell A, Debye and alpha relaxation strengths give almost identical curves that agree qualitatively with crystal fraction obtained in the MW fit, although the relaxation strength decreases faster than crystal fraction initially. In cell B, all three curves start out in the same way but separate later in the process, where both Debye and alpha relaxation strengths overestimate the degree of crystallinity. The MW crystallinity curve has a kink occurring approximately when there is a bump in the alpha relaxation strength, while this two-step behavior is not clearly seen in the Debye relaxation strength. The behavior of the crystallization process at $127.5 \mathrm{~K}$ and the MW analysis demonstrated in Fig. 5 are general for all the studied temperatures as can be seen in Fig. 6 .

The proposed MW analysis qualitatively and quantitatively agrees with using the decrease of the alpha relaxation strength rather than the Debye relaxation strength as a measure of the crystallinity of the sample. However, neither reflect the fact that sample does not crystallize fully. This is because both the Debye and the alpha processes vanish during crystallization, while only the beta process survives. The analysis does not account for that, a fact that is already clear from the fits in Figs. 5(a) and 5(b).

\section{Avrami analysis}

Another independent — and more routinely used - way of evaluating the crystal growth is through the Johnson-MehlAvrami-Kolmogorov (JMAK) equation originally developed by Avrami. ${ }^{34-36}$ In this analysis, the volume fraction taken
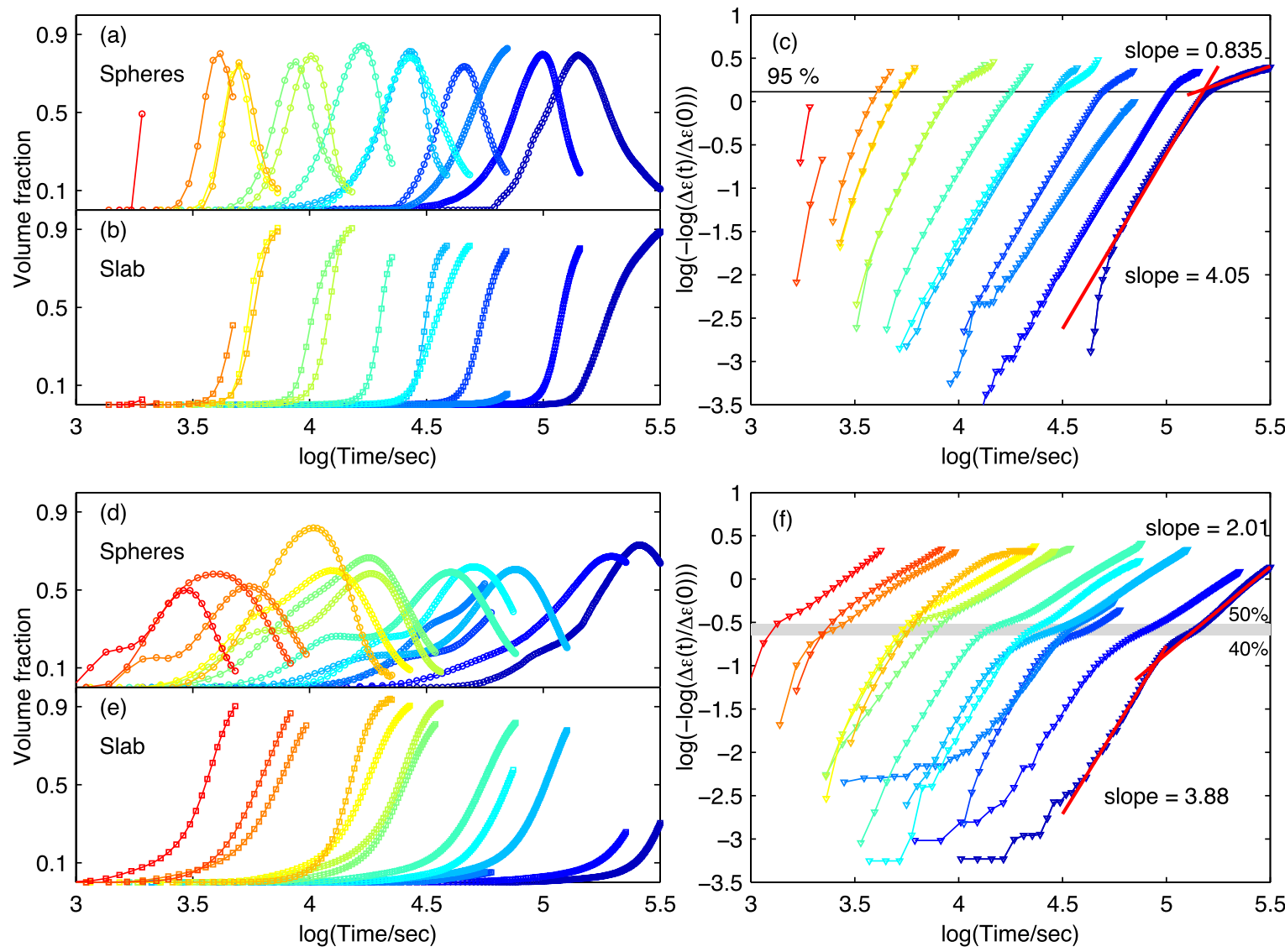

FIG. 6. Results from MW and Avrami analyses for all temperatures. (a), (b), (d), and (e) Volume fraction of crystal spheres (a) and (d) and volume fraction of a slab (b) and (d) based on the proposed combination of Eqs. (2) and (3). The differences for the two measuring cells outlined in Figs. 5(c) and 5(e) are general; cell A (a) and (b) display a single peak in the "sphere growth" and the "slab growth" set in early, while in cell B (d) and (e), there is a clear double peak in the "sphere growth." (c) and (f) show the JMAK plot which is a linearisation of the JMAK equation (Eq. (4) in the text). For both cells A (c) and B (f), the data clearly show a crossover from one linear behavior with a steep slope $(\sim 4)$ at short times to another linear behavior with a smaller slope $(\sim 1-2)$. But the transition from one to the other occurs at different crystallization degrees for the two cells: for cell A, the transition happens when the alpha relaxation strength has decayed more than $95 \%$ and for cell B, when $\Delta \epsilon(t) / \Delta \epsilon(0) \sim 40 \%-50 \%$ (marked by the gray bars in the figure). 
up by crystallites, $X_{c}$ is expressed in terms of a growth rate constant $k$, an induction time $t_{0}$, and the Avrami parameter $n$ as follows:

$$
X_{c}(t)=1-\exp \left[-k\left(t-t_{0}\right)^{n}\right]
$$

The value of $n$ depends on the crystal morphology and crystallization mechanism, but it is not straightforward to interpret the meaning of this parameter. Originally, it was a number between 1 and 4 such that $n=d+r$ with $d$ being the dimensionality of the growth and $r$ being a number that represents the nucleation rate. For a constant nucleation rate, $r=1$ and $r=0$ if nucleation stops when the crystallization starts. More recently, $n$ has been found to be a number between 1 and $7 .^{14,18}$

When dielectric spectroscopy is used to study crystallization, the common practice is to assume that the alpha relaxation strength roughly corresponds to the degree of crystallinity, $X_{c}$, and then use that for the Avrami analysis. Since the MW model also gives some support for the alpha rather than the Debye relaxation strength is expressing the degree of crystallinity in the sample, we will adopt this approach.

One way to obtain an estimate of the parameter $n$ is through the JMAK plot, which plots $\ln \left[-\ln \left(1-X_{c}(t)\right)\right]$ versus $\ln (t)$. This procedure avoids fitting Eq. (4) to data and $n$ is directly obtained as the slope of the curve. The JMAK plots for cells A and B are shown in Fig. 6. In both cases, we see a transition from a relatively high value $n \approx 4-5$ to a low value $n \approx 1-2$. This observation suggests a change from higher dimensionality of growth to lower, which is consistent with the MW analysis indicating a change from spherical to slab growth. Moreover, the data suggest that this transition happens earlier in the crystallization process for cell B compared to cell A, which could explain why the crystallization slows down and takes much longer in cell B.

\section{DISCUSSION}

Both the Debye and the alpha processes vanish during the crystallization, while the beta process survives. Thus, we confirm earlier findings that the crystallization process at temperatures near $T_{g}$ stops before the sample is completely crystallized. Hedoux et al. report signs of an aborted or frustrated crystallization process, signaled by an amorphous halo persisting in the x-ray spectra. ${ }^{25}$ This slow and frustrated crystallization process has also been interpreted as a polyamorph transformation between two meta-stable liquid phases. ${ }^{27,37-39}$ Based on the dielectric spectra presented here, it is perhaps difficult to distinguish between the two scenarios, but the fact that the structural relaxation peaks disappear entirely combined with the emergence of Bragg peaks as documented in Ref. 25 point to a non-trivial crystallization process as the most obvious explanation for the observations.

It is however interesting that the aborted crystallization is seen in the dielectric spectra as the survival of the beta-process. If we envision the end product as a frustrated crystal, unable to tile space, then the liquid signal - in our case the beta relaxation - could originate from small pockets of liquids between crystal grains. This picture supports the idea of the beta relaxation being a local phenomenon, in favor of the "islands of mobility" suggested by Johari and co-workers. ${ }^{40,41}$

The mono-hydroxyl alcohols in general are interesting because of their anomalous (and usually intense) relaxation process at frequencies lower than the structural alpha relaxation - the so called Debye relaxation — which is believed to be due to supra-molecular hydrogen bonded structures in the liquid. ${ }^{21,42}$ We observe that the Debye process vanishes faster than the alpha during crystallization, and that the alpha intensity seems to give a better measure for the degree of crystallinity. Sanz et al. $^{43}$ made similar observations for another monohydroxyl alcohol. They studied crystallization of isopropanol in real time by simultaneous dielectric spectroscopy and neutron diffraction measurements and thus had a direct measure of the degree of crystallinity that could be correlated with the relaxation strength of the Debye and alpha processes. They observed that the Debye intensity dropped rapidly at the onset of crystallization, while the alpha intensity followed the crystallization. Their intuitive and appealing interpretation was that the breakage of the hydrogen-bonded network is a precursor of the crystallization, and that the molecules leaving the network did not immediately go into a crystalline structure. The MW polarization effects lend itself to a different - macroscopic — interpretation of the observations. Irrespective of how the crystal growth is modeled in the MW framework, there cannot be proportionality between dielectric intensity and liquid fraction in the sample. The deviation from linearity depends on the specific model for the growth morphology and on the intensity of the process: the higher intensity, the stronger the deviation from linearity. Thus, the MW analysis provides a simple explanation for why the most intense process vanishes before the less intense one. The MW analysis does at the same account for the observed frequency shifts of the relaxation processes.

On the basis of MW fits, we suggested that the observed difference in crystallization behavior between the two cells could be rationalized by a transition from having primarily a growth of crystal spheres, a homogeneous nucleation and growth, to a growth of a crystal slab. This could be a slab growing from the electrodes but could also be a certain point in the process where crystal grains percolate and effectively create a crystal layer in the liquid-crystal mixture. The difference between the cells would then be explained by a difference in the degree of crystallization when this transition takes place. The idea that the crystal growth changes from a higher dimensional growth to a low dimensional growth was supported by the JMAK analysis that also points to such a transition taking place at different crystallization degrees in the two sample cells. The overall validity of JMAK equation (Eq. (4)) has been questioned, see, e.g., Refs. 44 and 45, and of course, we need to be cautious when making conclusions, based on the MW analysis where the limits of applicability of the mean-field approximations were pushed. But since both types of analyses point to this picture, we believe that the proposed conception of a change in morphology of the crystal growth is consistent and sound. It remains to be shown how general this behavior is. It would be interesting to apply this procedure to a simpler sample to study the influence of sample cell geometry on the crystallization process. 
Irrespective of the generality of the particular behavior found here, our study shows that one needs to be very cautious about making detailed microscopic interpretations of the crystallization mechanisms based on dielectric spectroscopy alone, because MW polarization effects of the mixed phase require knowledge about the crystal growth morphology. In addition, we have also shown that the crystallization is extremely sensitive to the specific sample environment. Thus, it would require extensive investigations of different environments and perhaps even different probes to disentangle microscopic from macroscopic effects.

\section{CONCLUSION}

We have studied the isothermal crystallization process in the deeply supercooled region of the mono-alcohol $n$-butanol in real time at 15 different temperatures using dielectric spectroscopy. Two different sample cells have been used to look for the effects of the sample environment on the crystallization process. We found that the time evolution of the relaxation strengths differs for the two cells in a consistent and reproducible way for all temperatures.

On the basis of the Maxwell-Wagner analysis, we suggest that the crystallization behavior can be explained by a transition from primarily growth of crystal spheres to growth of a crystal layer. The difference between the cells in this framework is the difference in when in the crystallization process this transition takes place. This picture was supported by an Avrami-Mehl-Johnson-Kolmogorov analysis that also suggests a transition from higher dimensional growth to a lower one.

The Maxwell-Wagner analysis can also account for the shift in peak frequency observed for the Debye process during the course of crystallization, and thus, a microscopic interpretation of the peak shift is not needed. In general, our study shows that any microscopic interpretation of crystallization measurements requires multiple probes, sample environments, and careful protocols.

\section{ACKNOWLEDGMENTS}

Tina Hecksher is sponsored by DNRF Grant No. 61. The authors thank Ranko Richert for useful discussions and valuable comments.

${ }^{1}$ G. Tammann, J. Soc. Glass Technol. 9, 166 (1925),

${ }^{2}$ W. Kauzmann, Chem. Rev. 43, 219 (1948).

${ }^{3}$ P. G. Debenedetti, Metastable Liquids : Concepts and Principles (Princeton University Press, Princeton, NJ, 1996).

${ }^{4}$ R. Becker and W. Döring, Ann. Phys. 416, 719 (1935).
${ }^{5}$ S. N. Bhat, A. Sharma, and S. V. Bhat, Phys. Rev. Lett. 95, 235702 (2005).

${ }^{6}$ O. Mishima and H. E. Stanley, Nature 396, 329 (1998).

${ }^{7}$ P. G. Debenedetti, J. Phys.: Condens. Matter 15, R1669 (2003).

${ }^{8}$ H.-F. Yuan, T. Xia, M. Plazanet, B. Deme, and M. Orrit, J. Chem. Phys. 136, 041102 (2012).

${ }^{9}$ J. Rabesiaka and A. J. Kovacs, J. Appl. Phys. 32, 2314 (1961).

${ }^{10}$ S. Napolitano and M. Wübbenhorst, Macromolecules 39, 5967 (2006).

${ }^{11}$ M. Massalska-Arodz, G. Williams, D. K. Thomas, W. J. Jones, and R. Dabrowski, J. Phys. Chem. B 103, 4197 (1999).

${ }^{12}$ J. Alie, J. Menegotto, P. Cardon, H. Duplaa, A. Caron, C. Lacabanne, and M. Bauer, J. Pharm. Sci. 93, 218 (2003).

${ }^{13}$ K. Adrjanowicz, K. Kaminski, Z. Wojnarowska, M. Dulski, L. Hawelek, S. Pawlus, M. Paluch, and W. Sawicki, J. Phys. Chem. B 114, 6579 (2010).

${ }^{14}$ A. K. R. Dantuluri, A. Amin, V. Puri, and A. K. Bansal, Mol. Pharm. 8, 814 (2011).

${ }^{15}$ K. Kothari, V. Ragoonanan, and R. Suryanarayanan, Mol. Pharm. 11, 3048 (2014).

${ }^{16}$ J. Sibik, M. J. Sargent, M. Franklin, and J. A. Zeitler, Mol. Pharm. 11, 1326 (2014).

${ }^{17}$ T. A. Ezquerra, J. Majszczyk, F. J. Balta-Calleja, L.-C. E., K. H. Gardner, and B. S. Hsiao, Phys. Rev. B 50, 6023 (1994).

${ }^{18}$ M. T. Viciosa, N. T. Correia, M. S. Sanchez, A. L. Carvalho, M. J. Romão, J. L. Gómez Ribelles, and M. Dionísio, J. Phys. Chem. B 113, 14209 (2009).

${ }^{19}$ K. Adrjanowicz, A. Grzybowski, K. Grzybowska, J. Pionteck, and M. Paluch, Cryst. Growth Des. 14, 2097 (2014).

${ }^{20}$ Broadband Dielectric Spectroscopy, edited by F. Kremer (Springer Science \& Business Media, 2003).

${ }^{21}$ R. Böhmer, C. Gainaru, and R. Richert, Phys. Rep. 545, 125 (2014).

${ }^{22}$ I. M. Shmyt'ko, R. J. Jiménez-Riobóo, M. Hassaine, and M. A. Ramos, J. Phys.: Condens. Matter 22, 195102 (2010).

${ }^{23}$ P. Derollez, A. Hedoux, Y. Guinet, F. Danede, and L. Paccou, Acta Crystallogr., Sect. B: Struct. Sci., Cryst. Eng. Mater. 69, 195 (2013).

${ }^{24}$ A. Wypych, Y. Guinet, and A. Hedoux, Phys. Rev. B 76, 144202 (2007).

${ }^{25}$ A. Hedoux, Y. Guinet, L. Paccou, P. Derollez, and F. Danede, J. Chem. Phys. 138, 214506 (2013).

${ }^{26}$ M. Hassaine and M. A. Ramos, Phys. Status Solidi A 208, 2245 (2011).

${ }^{27}$ R. Kurita and H. Tanaka, J. Phys.: Condens. Matter 17, L293 (2005).

${ }^{28}$ B. Igarashi, T. Christensen, E. H. Larsen, N. B. Olsen, I. H. Pedersen, T. Rasmussen, and J. C. Dyre, Rev. Sci. Instrum. 79, 045105 (2008).

${ }^{29}$ N. Saglanmak, A. I. Nielsen, N. B. Olsen, J. C. Dyre, and K. Niss, J. Chem. Phys. 132, 024503 (2010)

${ }^{30}$ A. I. Nielsen, B. Jakobsen, K. Niss, N. B. Olsen, R. Richert, and J. C. Dyre, J. Chem. Phys. 130, 154508 (2009).

${ }^{31}$ R. Richert, Eur. Phys. J.: Spec. Top. 189, 37 (2010).

${ }^{32}$ J. Schüller, R. Richert, and E. W. Fischer, Phys. Rev. B 52, 15232 (1995).

${ }^{33}$ K. W. Wagner, Arch. Elektrotech. 8, 371 (1914).

${ }^{34}$ M. Avrami, J. Chem. Phys. 7, 1103 (1939).

${ }^{35}$ M. Avrami, J. Chem. Phys. 8, 212 (1940).

${ }^{36}$ M. Avrami, J. Chem. Phys. 9, 177 (1941).

${ }^{37}$ A. G. Dzhonson and B. V. Bol'shakov, Dokl. Phys. Chem. 393, 318 (2003),

${ }^{38}$ B. V. Bol'shakov and A. G. Dzhonson, J. Non-Cryst. Solids 351, 444 (2005).

${ }^{39}$ B. Zgardzinska, M. Paluch, and T. Goworek, Chem. Phys. Lett. 491, 160 (2010).

${ }^{40}$ G. P. Johari and M. Goldstein, J. Chem. Phys. 53, 2372 (1970).

${ }^{41}$ G. P. Johari, J. Non-Cryst. Solids 307-310, 317 (2002).

${ }^{42}$ C. Gainaru, R. Figuli, T. Hecksher, B. Jakobsen, J. C. Dyre, and M. A. B. Wilhelm, Phys. Rev. Lett. 112, 098301 (2014).

${ }^{43}$ A. Sanz, M. Jiménez-Ruiz, A. Nogales, D. Martín y Marero, and T. A. Ezquerra, Phys. Rev. Lett. 93, 015503 (2004).

${ }^{44}$ M. T. Todinov, Acta Mater. 48, 4217 (2000).

${ }^{45}$ M. Fanfoni and M. Tomellini, Il Nuovo Cimento D 20, 1171 (1998). 